\title{
The Mobile Version of the Predicted Energy Efficient Bee-Inspired Routing (PEEBR)
}

\author{
Imane M. A. Fahmy, Hesham A. Hefny \\ ${ }^{1}$ Department of Computer Sciences \\ Institute of Statistical Studies and Research, \\ Cairo University \\ Giza, Egypt
}

\author{
Laila Nassef ${ }^{1,2}$ \\ ${ }^{2}$ Department of Computer Science \\ Faculty of Computing and Information Technology, \\ King Abdulaziz University \\ Jeddah, Saudi Arabia
}

\begin{abstract}
In this paper, the previously proposed Predictive Energy Efficient Bee-inspired Routing (PEEBR) family of routing optimization algorithms based on the Artificial Bees Colony (ABC) Optimization model is extended from a random static mobility model, as employed by its first version (PEEBR1), into a random mobility model in its second version (PEEBR2). This random mobility model used by PEEBR-2 algorithm is proposed and described. Then, PEEBR-2's was simulated in order to compare its performance relative to the first version (PEEBR-1) in terms of predicted optimal path energy consumption, nodes batteries residual power and fitness.

The simulation results have shown that PEEBR-2's optimal path is predicted to consume less energy and realizing higher fitness. On the other hand, PEEBR-1's optimal paths nodes possess higher batteries residual power. At last, the impact of mobile nodes speeds was studied for PEEBR-2 in terms of optimal path's predicted energy consumption and path nodes batteries residual power showing its performance stability relative to nodes mobility speed.
\end{abstract}

Keywords-PEEBR; PEEBR-1; PEEBR-2; Energy Efficient Routing; Bee-inspired; Artificial Bee Colony (ABC) optimization; Random Mobility Model

\section{INTRODUCTION}

Swarm Intelligence (SI) is a computational intelligence approach, as described by Mayur Tokekar and Radhika D. Joshi (2011) in [7] that is based on the study of collective behavior of social insects in decentralized, self-organized systems. SI involves a collective behavior of autonomous agents that locally interact with each other in a distributed environment to solve a given problem in the hope of finding a global solution to the problem as defined by J. Wang et al. (2009) in [8]. Ant Colony Optimization (ACO) introduced by M. Dorigo et al. (2006)in [9] and Artificial Bee Colony (ABC) Optimization by D. Karaboga and B. Basturk (2007) in [1-3] are among SI optimization techniques that are relatively more robust, reliable, and scalable than other conventional routing algorithms.

The Artificial Bee Colony (ABC) Optimization model introduced by D. Karaboga and B. Basturk (2007) was a general purpose swarm Intelligence SI optimization technique based on efficient labor employment and efficient energy consumption through a multi-agent distributed model. The Predictive Energy Efficient Bee-inspired Routing (PEEBR) was inspired from the honey bees foraging behavior based on the natural bees food source search behavior that aims to discover, exploit and direct the swarm of working bees into the highest quality food source. It tends to map $\mathrm{ABC}$ 's optimization model equations onto Mobile Ad-hoc wireless Network MANET routing parameters used to evaluate potential paths between a certain source and destination. Therefore, PEEBR could be considered as a power-cost efficient algorithm that uses two types of bee agents to collect information about every potential path from source to destination then evaluate their efficiency and assign an equivalent fitness value and goodness ratio for each potential path based on the number of hops, the amount of energy to be consumed and the path nodes residual battery energy.

The proposed Predictive Energy Efficient Bee-inspired Routing mobile version (PEEBR-2) is the second version of PEEBR considering nodes mobility while routing. The critical mobility parameter represented by the mobile nodes speed's effect on average predicted energy consumption by the optimal path nodes and on the optimal path nodes battery residual power while routing using PEEBR-2 algorithm will be shown and analyzed in this chapter.

This paper is organized as follows: the second section presents the Predictive Energy Efficient Bee-inspired Routing1: PEEBR-1 algorithm and model. Then, PEEBR-1's path selection mechanism is discussed by the third section. The fourth Section will propose the mobile version PEEBR-2 mobility model and algorithm. PEEBR-2's simulation results are discussed by the fifth section and the paper's contribution is summarized by the conclusion in the sixth section.

\section{PEEBR-1 Algorithm AND MOdEL}

The proposed Predictive Energy-Efficient Bee Routing-1 (PEEBR-1), proposed by Imane M. A. Fahmy et al. 2012 [4] then improved and evaluated in [5-6], is assumed to be a reactive routing algorithm that enables a source node to discover the optimal path to a destination node based on the expected energy to be consumed during packets reception and the path nodes residual battery power.

\section{A. Mapping ABC Model onto PEEBR}

In the following table 1 , the inspired $A B C$ model's elements are mapped to the PEEBR's algorithm elements together with their optimization interpretation in order to clarify the inspired parts of the ABC model including: The fitness function and the probability associated with each potential path. 
TABLE I. MAPPING ABC MODEL ONTO PEEBR ALGORITHM's OPTIMIZATION PARAMETERS

\begin{tabular}{|c|c|c|}
\hline $\begin{array}{l}\text { Optimizatio } \\
\text { n Algorithm }\end{array}$ & $\mathbf{A B C}$ & PEEBR \\
\hline $\begin{array}{l}\text { Possible } \\
\text { solution to } \\
\text { optimize }\end{array}$ & Food Source Position & $\begin{array}{l}\text { Path between a } \\
\text { source node \& } \\
\text { destination }\end{array}$ \\
\hline $\begin{array}{l}\text { Solution } \\
\text { quality }\end{array}$ & Amount of nectar & $\begin{array}{l}\text { Average path nodes } \\
\text { residual power }\end{array}$ \\
\hline $\begin{array}{l}\text { Number of } \\
\text { solutions }\end{array}$ & Number of employed bees & $\begin{array}{l}\text { Number of potential } \\
\text { paths }\end{array}$ \\
\hline $\begin{array}{l}\text { Cost } \\
\text { Function }\end{array}$ & $\begin{array}{l}f_{i} \\
=\frac{1}{D_{\text {Train }}} \sum_{j=1}^{D_{\text {Train }}} d\left(x_{j}, P_{i}^{C L_{\text {Known }}\left(x_{j}\right)}\right)\end{array}$ & $\begin{array}{l}f\left(R_{j}\right) \\
=h\left(R_{j}\right) \sum_{i=1}^{N j} \frac{E_{r}\left(n_{i j}\right)}{B\left(n_{j i}\right)}\end{array}$ \\
\hline $\begin{array}{l}\text { Fitness } \\
\text { Function }\end{array}$ & $f i t_{i}=\frac{1}{1+f_{i}}$ & $\begin{array}{l}\text { fit }\left(R_{j}\right) \\
=\frac{1}{1+f\left(R_{j}\right)}\end{array}$ \\
\hline $\begin{array}{l}\text { Number of } \\
\text { training } \\
\text { patterns }\end{array}$ & $D_{\text {Train }}$ & $\mathrm{N}$ nodes \\
\hline $\begin{array}{l}\text { Number of } \\
\text { Solution }\end{array}$ & SN Sources Number & M paths \\
\hline $\begin{array}{l}\text { Probability of } \\
\text { solution }\end{array}$ & $P_{i}=\frac{f i t_{i}}{\sum_{n=1}^{S N} f i t_{n}}$ & $\begin{array}{l}\mathrm{G}\left(\mathrm{R}_{\mathrm{j}}\right) \\
=\frac{f i t\left(R_{j}\right)}{\sum_{\mathrm{j}=1}^{\mathrm{M}} f i t\left(R_{j}\right)}\end{array}$ \\
\hline
\end{tabular}

By selecting the path that consumes less energy and hence reserve the nodes batteries along the path, this will certainly extend the network lifetime by extending the nodes batteries lifetime.

\section{B. PEEBR-1 Model}

In figure 1 , there are three potential routing paths from the source node "A" towards the destination node "J": the first potential path R1: A, B, E, J, the second potential path R2: A, C, F, J, and finally, the third path R3: A, D, G, I, J.

The selection of the optimal path among these three paths depends on the amount of energy expected to be consumed by the mobile nodes over that path during communication. This energy information will be collected by the forward bee agents during path discovery journey and includes mainly two essential metrics: the first is nodes battery power residual to determine their expected lifetime and hence their efficiency in relaying the data packets along the path. While the second is the total amount of energy expected to be consumed by all nodes along the path for each path represented by $E\left(R_{j}\right)$ where $\mathrm{j}$ is the path index.

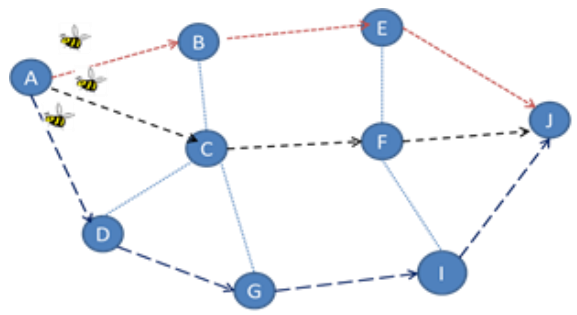

Fig. 1. An example of path discovery process using bee agents

It is noteworthy to mention that the first objective for routing optimization is minimizing the amount of energy consumed during the communication. In order to calculate the total amount of energy consumed by all the nodes along each path during reception is given by the general expression (1) that could be applied for the former example on three possible paths: R1, R2 and R3.

$$
E(R j)=h(R j) * E_{r}(p)
$$

Where $h(R j)$ is the number of hops over a path $R j$ and $E_{r}(p)$ is the amount of energy consumed during reception of a packet $\mathrm{p}$.

If $\boldsymbol{E}(\boldsymbol{R} \mathbf{1})<\boldsymbol{E}(\boldsymbol{R} 2)<\boldsymbol{E}(\boldsymbol{R} \mathbf{3})$ then R1 is the routing path that achieves the least power consumption in case it meets all other threshold constraints such as: the nodes battery residual power $\mathrm{P}(\mathrm{n})$ that enables reliable data packets transmission during communication along the path and the then R1: A, B, E, J will be selected as the optimal path between the source node $\mathrm{A}$ and the destination node $\mathrm{J}$.

Therefore, the path goodness ratio $g\left(\mathrm{R}_{\mathrm{j}}\right)$ could be a ratio assigned to each potential path from $A$ to $J$ reflecting the path quality combining: the total amount of energy expected to be consumed by the path's nodes, the path nodes residual battery energy $\boldsymbol{B}\left(\boldsymbol{n}_{\boldsymbol{j} \boldsymbol{i}}\right)$ and the number of hops $\boldsymbol{h}\left(\boldsymbol{R}_{\boldsymbol{j}}\right)$.

The second objective for routing optimization is maximizing the battery residual power of the selected path's nodes $\mathrm{B}\left(\mathrm{R}_{\mathrm{j}}\right)$

In order to achieve both objectives: the least power consumption and selecting the path with the maximum nodes battery residual energy, these objectives could be combined in a cost function that aims to minimize energy consumption and maximize battery residual power as given by expression (2).

$$
f\left(R_{j}\right)=h\left(R_{j}\right) \sum_{i=1}^{N_{j}} \frac{E\left(n_{i j}\right)}{B\left(n_{i j}\right)}
$$

Finally, the path fitness $\boldsymbol{f} \boldsymbol{i t}\left(\boldsymbol{R}_{\boldsymbol{j}}\right)$ relative to all potential M paths is represented by the general expression (3):

$$
\text { fit }\left(R_{j}\right)=\frac{1}{1+f\left(R_{j}\right)}
$$

Each potential path between the source node and the destination node could be represented by a goodness ratio reflecting its energy consumption and its nodes battery residual energy. The probability of a path selection $\mathbf{G}\left(\mathbf{R}_{\mathbf{j}}\right)$ reflecting its goodness ratio could be computed by expression (4):

$$
\mathrm{G}\left(R_{j}\right)=\frac{f i t\left(R_{j}\right)}{\sum_{\mathrm{j}=1}^{\mathrm{M}} \text { fit }\left(R_{j}\right)}
$$

In order to test PEEBR's performance, it was run on $\mathrm{T}_{\max }=100$ iterations. The nodes battery residual power $\mathrm{B}\left(\mathrm{n}_{\mathrm{ij}}\right)$ was decreased after each iteration to reflect the real world's battery power decay as given by expression (5):

$$
B\left(n_{i j}\right)=B\left(n_{i j}\right)^{0} * e^{\frac{-t}{\tau}}
$$

Where $B\left(n_{i j}\right)^{0}$ is the initial node battery residual power, $\mathbf{t}$ is the iteration number and $\boldsymbol{\tau}$ is a time constant. Finally, PEEBR-1 termination conditions were: reaching the maximum number of iterations $\mathrm{T}_{\max }$ or a minimal predefined fitness value. The candidate path solution $\mathbf{R}_{\mathbf{c}}$ is the path with the highest goodness ratio deduced at the end of each iteration (It): $\mathbf{R}_{\mathrm{c}}=\mathbf{R}_{\mathrm{o}}[\mathrm{It}]$ 
Finally the optimal path $\mathbf{R}_{\mathbf{o}}$ is the optimal path deduced before one of the stopping conditions (The maximum number of iterations $T_{\max }$ or the Minimum Goodness Ratio MGR) is reached that is:

$$
\mathrm{R}_{\mathrm{o}}=\arg \max _{\mathrm{j}}\left\{\mathrm{G}\left(\mathrm{R}_{\mathrm{j}}\right)\right\}
$$

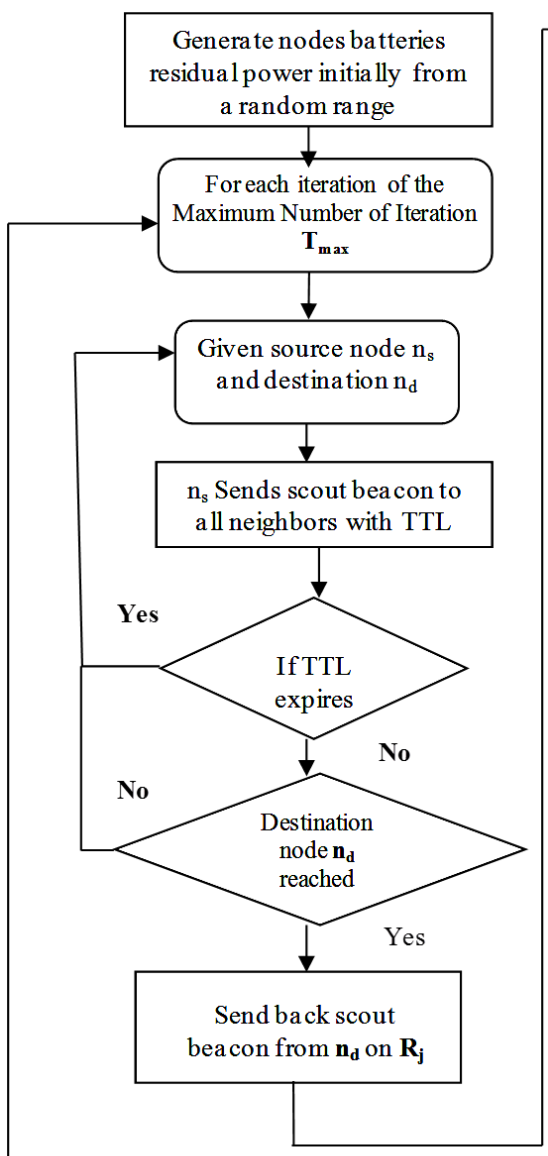

$\mathrm{m}$

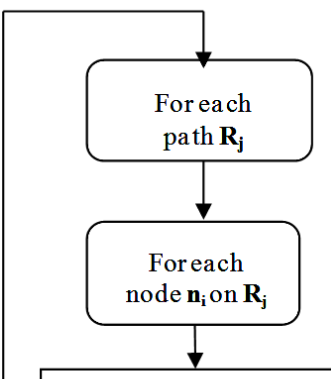

Foragers Compute consumed energy $\mathbf{E}_{\mathbf{r}}\left(\mathbf{n}_{\mathbf{i}}\right)$

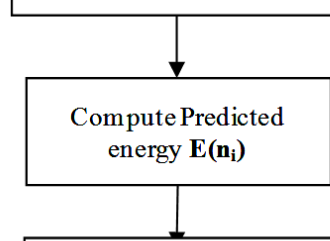

Foragers Compute energy and residual battery $\mathbf{B}\left(\mathbf{R}_{\mathbf{j}}\right)$

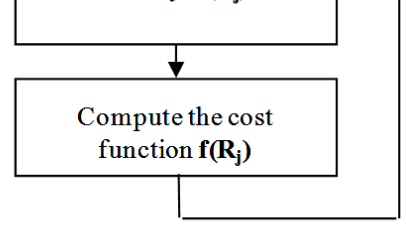

\section{PEEBR-1 PATH SELECTION MECHANISM}

The proposed predictive energy efficient model inspired by the BCO swarm intelligent model is an optimization model for the MANET.

\section{A. PEEBR-1 FLOW CHART}

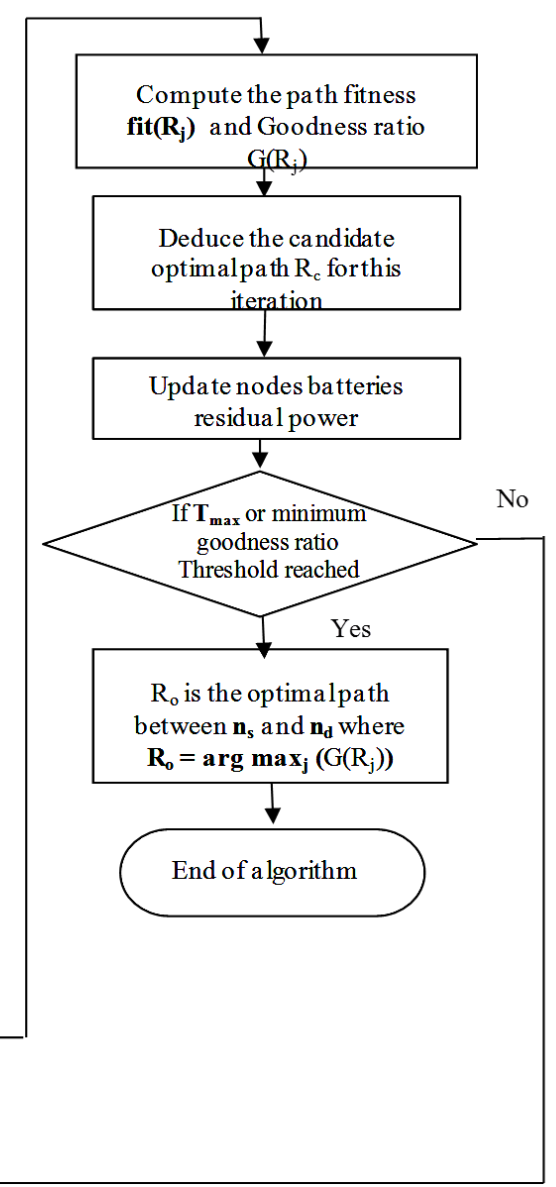

Fig. 2. PEEBR-1 Iterative Optimization Algorithm Flow Chart

\section{B. PEEBR-1 ALGORITHM}

PEEBR-1 algorithm for the optimal candidate path discovery process from source $n_{s}$ to the destination node $n_{d}$ could be summarized by algorithm 1 .

Algorithm 1: The Predictive Energy-Efficient Bee Routing-1 (PEEBR-1)

\section{PEEBR-1(Net_Topology, $\left.\mathrm{n}_{\mathrm{s}}, \mathrm{n}_{\mathrm{d}}, M G R\right)$} //The Scout bee phase:

1. Source node $\mathbf{n}_{\mathbf{s}}$, floods a "Scout packet" associated with a Time-To-Live TTL to all $\mathbf{N}_{\mathbf{j}}$ neighboring nodes on all $\mathbf{M}$ potential paths.

2. Each "Scout" $\mathbf{j}$ flies over one of the $\mathbf{M}$ potential routes $\mathbf{R}_{\mathbf{j}}$ until it reaches destination node $\mathbf{n}_{\mathbf{d}}$.

3. If $(\mathbf{T T L}=\mathbf{= 0})$

expires over a neighboring path
Then "Scout" bee agent packet will die indicating failure to reach destination to the source and the corresponding routing path will be avoided.

4. When a bee agent reaches the destination node $\mathbf{n}_{\mathbf{d}}$, it is sent back to its source $\mathbf{n}_{\mathbf{s}}$ through the same traveled route.

5. The "Scout packet" collects the potential route's routing information including:

- Count number of hops $\mathbf{h}\left(\mathbf{R}_{\mathbf{j}}\right)$

- Collect each route nodes residual battery power $\mathbf{B}\left({ }_{\text {nji }}\right)$ where $\mathbf{i}=1$ to $\mathbf{N}_{\mathbf{j}}$ nodes

- Collect the amount of predicted receiving power $\mathbf{E}_{\mathbf{r}}(\mathbf{R} \mathbf{j})$

\section{//The Forager bee phase:}

6. At the source node $\mathbf{n}_{\mathbf{s}}$, the "forager" evaluation process starts by calculating the predicted amount of energy to be consumed for each "Backward Scout" discovered route using (1). 
7. Each potential route cost $\boldsymbol{f}\left(\mathbf{R}_{\mathbf{j}}\right)$ is calculated for each route $\mathbf{R j}$ dependent on its hop count $\mathbf{h}\left(\mathbf{R}_{\mathbf{j}}\right)$, its nodes residual battery power $\mathbf{B}\left({ }_{\mathrm{nji}}\right)$ and its expected amount of receiving power consumed $\mathbf{E}_{\mathbf{r}}(\mathbf{R j})$ using (2).

8. Associate a fitness value reflecting the goodness of each route $\boldsymbol{f i t}\left(\mathbf{R}_{\mathbf{j}}\right)$ using (3).

9. Assign each route a goodness ratio $\mathbf{G}\left(\mathbf{R}_{\mathbf{j}}\right)$ using (4)

10. If $\left(\mathbf{G}\left(\mathbf{R}_{\mathbf{o}}\right)<\right.$ MGR $)$ Then exit //stopping criteria for minimum goodness rati

11. Get the candidate path solution $\mathbf{R}_{\mathbf{c}}=\mathbf{R}_{\mathbf{o}}$ [It] between $\mathbf{n}_{\mathbf{s}}$ and $\mathbf{n}_{\mathbf{d}}$ is the route with the maximum goodness as given by (5)

Therefore, PEEBR-1 algorithm for the optimal candidate path discovery process from source $\mathrm{n}_{\mathrm{s}}$ to the destination node $\mathrm{n}_{\mathrm{d}}$ could be summarized by algorithm 2 .

Algorithm 2: The Iterative Predictive Energy-Efficient Bee Routing-1 (PEEBR-1) Optimization

1. Choose one of twenty randomly generated MANET topologies. //select Net_Topology

2. Generate randomly nodes batteries residual power from a pre-defined range.

3. Define a Maximum Number of Iterations $\mathbf{T}_{\max }$ to run the following phases.

4. Define a Minimum Goodness Ratio MGR threshold.

5. Define a Time-To-Live TTL for the "Scout packet".

6. Input source and destination nodes $\mathbf{n}_{\mathbf{s}}$ and $\mathbf{n}_{\mathbf{d}}$

7. For (iteration $\mathrm{e}=1 ; \mathrm{e}<=\mathbf{T}_{\max } ; \mathrm{e}++$ )

PEEBR-1(Net_Topology, $\mathrm{n}_{\mathrm{s}}, \mathrm{n}_{\mathrm{d}}, M G R$ )

8. Update routes nodes batteries residual power for next iteration using (5-10)

9. $\quad$ End

10. Get the optimal route $\mathbf{R}_{\mathbf{0}}$ between $\mathbf{n}_{\mathbf{s}}$ and $\mathbf{n}_{\mathbf{d}}$ with the maximum goodness as given by (5-11)

\section{The Proposed PEEBR-2 Model AND Algorithm}

The proposed Predictive Energy Efficient Bee-inspired Routing (PEEBR-2) mobility model, flow chart and algorithm are described by the following sub-sections.

\section{A. The Proposed PEEBR-2 Mobility Model}

The random mobility model used by PEEBR-2 is based on MANET topologies variation. Each MANET topology Net (k) is formed according to expression (7):

$\operatorname{Net}(k)=\{(x(i, k), y(i, k)), 1 \leq i \leq N, 1 \leq k \leq L\}$

Where $\mathbf{i}$ is the mobile node ID on MANET topology $\mathbf{k}$. Moreover, $\mathbf{N}$ is the number of nodes (e.g. $\mathbf{N}=25$ nodes in the studied mobility scenarios) and $\mathbf{L}$ is the number of random topologies generated for the mobility model (e.g. L $=20$ random topologies).
Then, the random topology variation from time interval to another could be computed as given by expression (8) as follows:

$$
\Delta N e t(k)=\{(\Delta x(i, k), \Delta y(i, k)), 1 \leq i \leq N, 1 \leq k \leq L\}
$$

The random mobility model used by PEEBR-2 is based on the mobile nodes positions random variation. Assuming that every mobile node is moving in a circle of the MANET area with radius $\mathbf{r}_{\mathbf{k}}$ with a random angle $\boldsymbol{\theta}_{\mathbf{k}}$, the initial nodes coordinates for the first MANET topology are given by equations (9) and (10):

$x(i, k)=r_{k} * \cos \theta_{k}$

$y(i, k)=r_{k} * \sin \theta_{k}$

Where the random angle is within the range: $\boldsymbol{\theta}_{\mathbf{k}} \mathbf{\epsilon}[\mathbf{0 , 2 \pi}]$ and $\mathbf{r}_{\mathbf{k}} \boldsymbol{\epsilon}\left[\mathbf{0}, \mathbf{r}_{\max }\right]$ is the mobile node's displacement distance limited by its maximum displacement distance $\mathbf{r}_{\max }$. Then, the next random topologies, the new mobile nodes coordinates are deduced from equations (11) and (12):

$$
\begin{aligned}
& \Delta x(i, k+1)=x(i, k)+\left(r_{k} * \cos \theta_{k}\right) \\
& \Delta y(i, k+1)=y(i, k)+\left(r_{k} * \sin \theta_{k}\right)
\end{aligned}
$$

Therefore, the next MANET topology could be generated from the sum of the previous and its variation using expression (13):

$$
\operatorname{Net}(k+1)=\operatorname{Net}(k)+\Delta N e t(k)
$$

The pre-defined (constant or within a random range) mobile node's displacement distance $\mathbf{r}$ in meters and the input mobility speed $\mathbf{v}$ in meters/second are used to compute the time $t$ in seconds for each random topology using equation (14):

$$
t=\frac{r}{v}
$$

The mobility simulation parameters data are generated from mobility simulation software based on the former expressions. Then, each MANET mobility topology represents a time interval. Within the same time interval, PEEBR-2 runs for the pre-defined number of iterations. Afterwards, at end of each topology, the computed time $\mathbf{t}$ is used to update the potential paths nodes batteries residual power for the next time interval.

\section{B. PEEBR-2 Flow Chart and Algorithm}

The proposed Predictive Energy Efficient Bee-inspired Routing for mobility mode: PEEBR-2 for the MANET is based on applying the random mobility model. It could be summarized as shown by the flow chart in figure 3. Then PEEBR-2's detailed algorithm will be discussed by algorithm (3). 


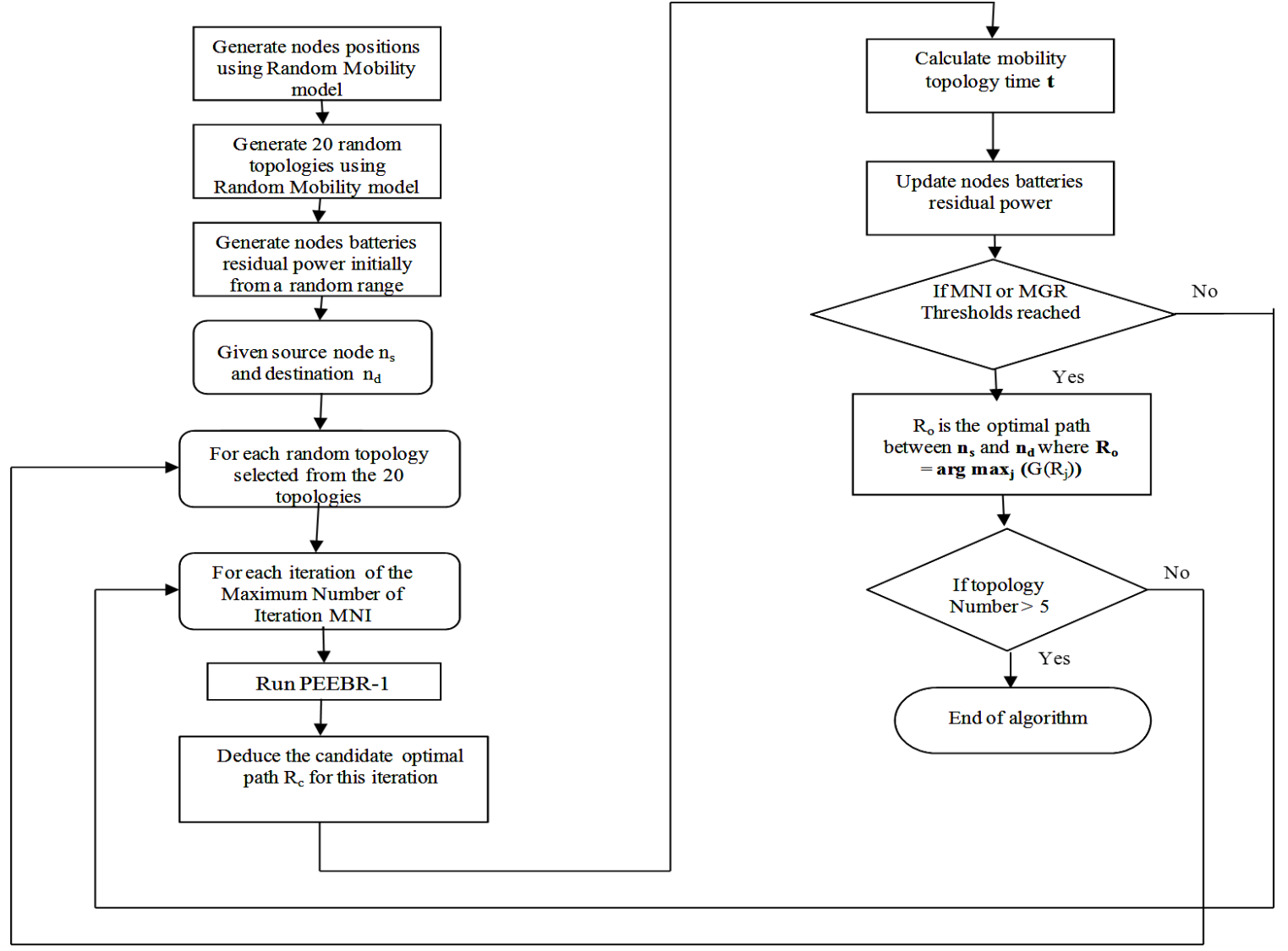

Fig. 3. PEEBR-2 Iterative Optimization Algorithm Flow Chart

Therefore, PEEBR-2 algorithm for the optimal candidate path discovery process from source $\mathrm{n}_{\mathrm{s}}$ to the destination node $\mathrm{n}_{\mathrm{d}}$ could be summarized by algorithm 3 .

Algorithm 3: The Predictive Energy Efficient Bee-inspired Routing-2: (PEEBR-2)

$\operatorname{PEEBR-2}\left(\mathbf{v}, \mathbf{r}_{\mathrm{k}}, \boldsymbol{\theta}_{\mathrm{k},} \mathbf{n}_{\mathrm{s}}, \mathbf{n}_{\mathrm{d}}\right)$

For (iteration $\mathrm{It}=1$; It $<=\mathrm{MNI}$; It ++ ) $/ / \mathrm{MNI}$ is

Maximum Number of Iterations

- PEEBR-1(Net_Topology, $\left.\mathrm{n}_{\mathrm{s}}, \mathbf{n}_{\mathrm{d}}, M G R\right)$

- Deduce $\mathbf{R}_{\mathbf{C}}$ the candidate optimal path between $\mathbf{n}_{\mathbf{s}}$ and $\mathbf{n}_{\mathbf{d}}$ is the route with the maximum goodness as given by (5)

- Calculate the topology mobility time $\mathbf{t}$ given the input mobile node displacement $\mathbf{r}_{\mathbf{k}}$ and speed $\mathbf{v}$ using (14)

- Update the potential routes nodes batteries residual power using (4) as function of the mobility time $\mathbf{t}$.

- Return the candidate path $\mathbf{R}_{\mathbf{c}}=\mathbf{R}_{\mathbf{o}}[$ It $]$ from $\mathbf{n}_{\mathbf{s}}$ to

$$
\mathbf{n}_{\mathbf{d}}
$$

End

End

Hence, algorithm 4 presents PEEBR-2 iterative optimization algorithm for optimal path discovery process between source $n_{s}$ to the destination node $n_{d}$.
Algorithm 4: The Iterative Energy Efficient Bee-inspired Routing-2 (PEEBR-2) Optimization

- Generate randomly nodes batteries residual power from a pre-defined range.

- Define a Maximum Number of Iterations MNI to run the following phases.

- Define a Minimum Goodness Ratio MGR threshold.

- Define a Time-To-Live TTL for the "Scout packet".

- Generate an initial MANET topology with nodes coordinates using (7).

- Generate twenty random MANET mobility topologies using (13).

- $\quad$ Set Simulation Time ST

- Choose five random Network Topologies NT each representing a time interval from the twenty generated topologies

- $\quad$ Input source and destination nodes $\mathbf{n}_{\mathbf{s}}$ and $\mathbf{n}_{\mathbf{d}}$

- $\quad$ For (Network Topology NT =1; NT<=5; NT++)

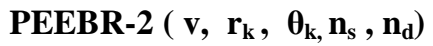

- Get the optimal route $\mathbf{R}_{\mathbf{o}}$ between $\mathbf{n}_{\mathbf{s}}$ and $\mathbf{n}_{\mathbf{d}}$ that is the route with the maximum goodness according to expression (5). 


\section{PEEBR-2 SimULATION RESULTS}

The experiment is based on optimal path selection between the arbitrary source and destination nodes for both protocols. PEEBR-2 is working on first random mobility scenario with 150 meters displacement distance (i.e., $r=150 \mathrm{~m}$ ).

In the following sub-sections, PEEBR-1 is compared to PEEBR-2 for different MANET sizes (number of nodes) in order to evaluate the impact of mobility with number of nodes variation on PEEBR's algorithm performance.

\section{A. Optimal Path Predicted Energy Consumption}

In figure 4, PEEBR-1's and PEEBR-2 are compared in terms of optimal path predicted energy consumption (joules) for different MANET sizes.

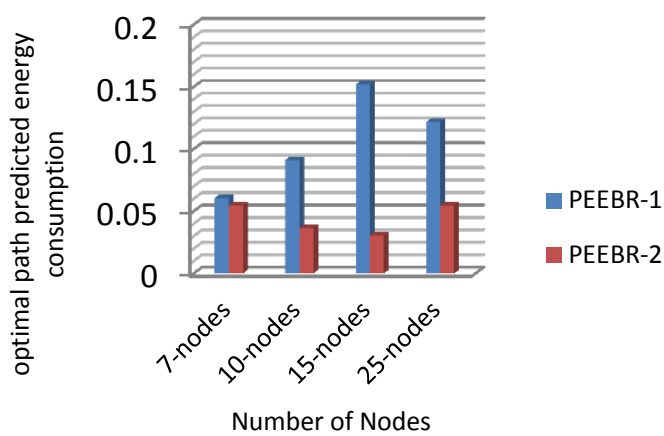

Fig. 4. Impact of number of nodes on optimal path average energy consumption

As shown by figure 4, the mobile PEEBR-2 outperforms the static PEEBR-1 for different MANET sizes from small to medium sizes since it results in less energy consumption.

\section{B. Optimal Path Nodes Batteries Residual Power}

In figure 5, PEEBR-1's and PEEBR-2 are compared in terms of optimal path nodes batteries residual power (joules) for different MANET sizes.

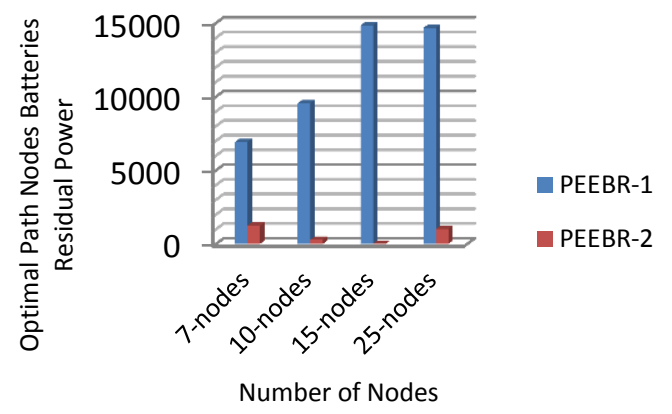

Fig. 5. Impact of number of nodes on optimal path average nodes batteries residual power

As shown by figure 5, PEEBR-1's optimal path nodes batteries residual power is higher and outperforms PEEBR-2 for all different MANET sizes (7-nodes, 10-nodes, 15-nodes and 25-nodes) especially for medium size MANETs (15- nodes) since mobility results nodes batteries power exponential degradation.

\section{Optimal Path Fitness}

In figure 6, PEEBR-1's and PEEBR-2 are compared in terms of optimal path fitness for different MANET sizes.

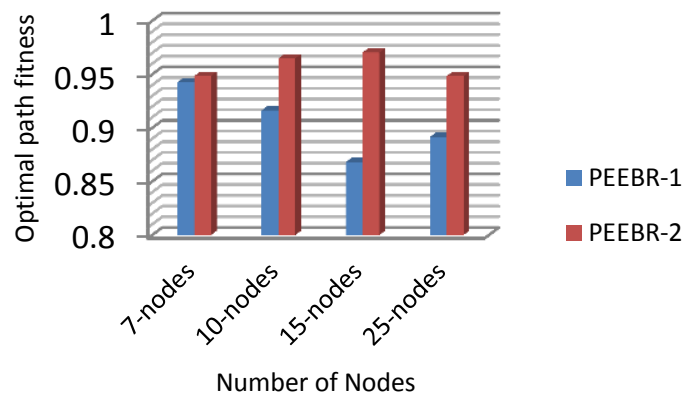

Fig. 6. Impact of number of nodes on optimal path average fitness

As deduced from figure 6, PEEBR-2's optimal paths achieved higher optimal path fitness. The static PEEBR-1's optimal path fitness is clearly lower since it is inversely proportional to the path cost. Hence, PEEBR-1 resulted in higher cost paths relative to the mobile PEEBR-2 for all different MANET sizes (7-nodes, 10-nodes, 15-nodes and 25nodes) especially for medium size MANETs (15-nodes).

\section{Impact of Mobile Nodes Speed Variation}

The impact of the mobile nodes speed variation is studied in terms of the optimal path energy consumption and batteries residual power.

\section{1) Optimal Path Predicted Energy Consumption}

In figure 7, PEEBR-2's predicted energy consumption firstly decreased with mobile nodes speeds from 10 to 20 $\mathrm{m} / \mathrm{sec}$, then, it maintained a stable consumption for speeds in the range from 30 to $50 \mathrm{~m} / \mathrm{sec}$.

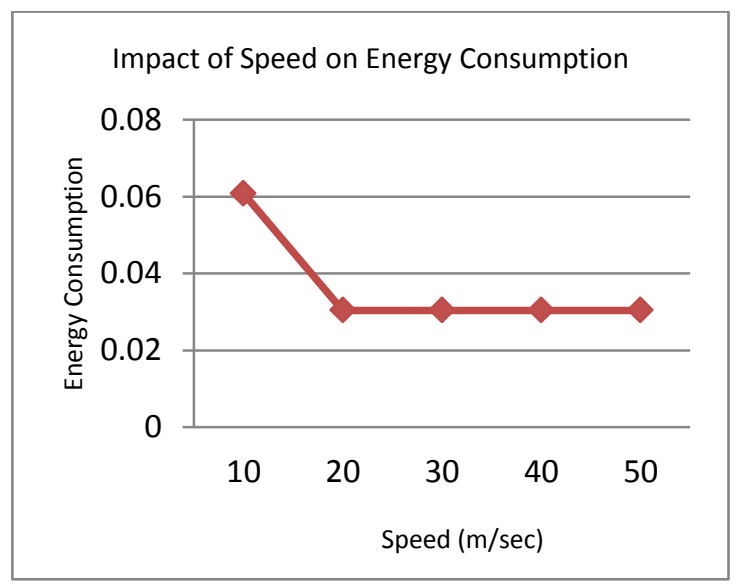

Fig. 7. Impact of mobile nodes speed on optimal path predicted energy consumption

2) Optimal Path Nodes Batteries Residual power

In figure 7, PEEBR-2's optimal path nodes batteries residual power decreased with mobile nodes speeds from 10 to 
$20 \mathrm{~m} / \mathrm{sec}$, then, it maintained a nearly constant path nodes batteries residual power for speeds in the range from 30 to 50 $\mathrm{m} / \mathrm{sec}$.

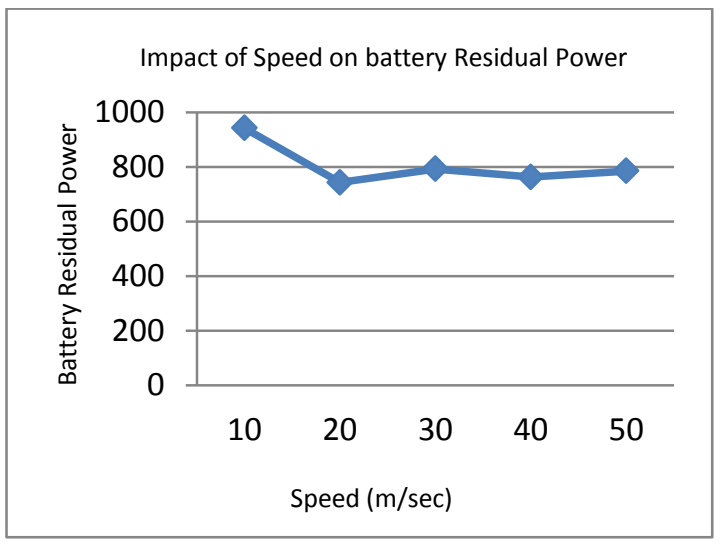

Fig. 8. Impact of mobile nodes speed on optimal path nodes batteries residual power

\section{CONCLUSION AND FUTURE WORK}

The previously proposed Predictive Energy Efficient Beeinspired Routing-1 (PEEBR-1) algorithm and flow chart were presented after model improvements. Then, the mobile version of the Predictive Energy Efficient Bee-inspired Routing-2 (PEEBR-2) algorithm and flow chart were introduced considering MANET nodes random mobility.

Then, PEEBR-2 algorithm's random mobility model was proposed. Its performance evaluation was performed using simulation in order to compare it with (PEEBR-1) in terms of the predicted optimal path predicted energy consumption, the optimal path nodes batteries residual power and the optimal path fitness.

The simulation experiments showed that PEEBR-2 outperformed PEEBR-1 in terms of optimal path predicted energy consumption and fitness. However, PEEBR-1's optimal paths attained higher batteries residual power.
Finally, the impact of mobile nodes speeds was assessed by means of optimal path's predicted energy consumption and path nodes batteries residual power revealing a stable performance.

The future work of this research work aims to test and evaluate PEEBR-2 performance against different performance parameters such as time and overhead delay.

\section{REFERENCES}

[1] D. Karaboga and B. Basturk (2007) 'A powerful and efficient algorithm for numerical function optimization: artificial bee colony (ABC) algorithm', Journal of Global Optimization, Springer, Vol. 39, No. 3, pp. 459-471.

[2] D. Karaboga and B. Basturk (2008), 'On the performance of artificial bee colony (ABC) algorithm', Elsevier, Applied Soft Computing, Vol. 8, pp.687-697.

[3] D. Karaboga and Ozturk (2011), 'A novel clustering approach: Artificial Bee Colony (ABC) algorithm', Elsevier, Applied Soft Computing, Vol. 11, pp. 652-657.

[4] Imane M. A. Fahmy, Laila Nassef and Hesham A. Hefny (2012), 'PEEBR: Predictive Energy Efficient Bee Routing Algorithm for Adhoc Wireless Mobile Networks', IEEE INFOrmatics and Systems (INFOS2012)Conference, Computer Networks Track, NW pp. 18-26.

[5] Imane M. A. Fahmy, Laila Nassef, Hesham A. Hefny 2014 (1), 'On the Performance of the Predicted Energy Efficient Bee-Inspired Routing (PEEBR)', IJACSA International Journal of Advanced Computer Science and Applications, Vol. 5, No. 4, pp.65-70.

[6] Imane M. A. Fahmy, Laila Nassef, Hesham A. Hefny 2014 (2), 'The Predicted Energy Efficient Bee-inspired Routing (PEEBR) Improvement and Performance Evaluation', In Proceedings of 18th international conference on Circuits, Systems, Communications and Computers (CSCC 2014), Santorini Island, Greece, July 17-21, 2014, Latest Trends on Communications, ISBN: 978-1-61804-235-4, pp.182-189.

[7] Mayur Tokekar and Radhika D. Joshi (2011), 'Enhancement of Optimized Linked state routing protocol for energy conservation', CCSEA 2011, CS \& IT 02, pp. 305-319.

[8] J. Wang, E. Osagie, P. Thulasiraman, R. K. Thulasiram (2009), 'HOPNET: A Hybrid ant colony OPtimization routing algorithm for Mobile ad hoc NETwork', Elsevier Ad Hoc Networks, Vol.7, No. 4, pp. 690-705.

[9] M. Dorigo, M. Birattari and Thomas Stutzle (2006), 'Ant Colony Optimization: Artificial Ants as computational intelligence technique', Université libre de Bruxelles, Belgique IEEE Computational Intelligence magazine, Vol.1, No. 4, pp. 28-39 